\title{
Using the UK Biobank as a global reference of worldwide populations: application to measuring ancestry diversity from GWAS summary statistics
}

\author{
Florian Privé ${ }^{1}$
}

${ }^{1}$ National Centre for Register-based Research, Aarhus University, Aarhus, 8210, Denmark.

Contact:

- florian.prive.21@gmail.com

\begin{abstract}
The UK Biobank project is a prospective cohort study with deep genetic and phenotypic data collected on almost 500,000 individuals from across the United Kingdom. Within this dataset, we carefully define 21 distinct ancestry groups from all four corners of the world. These ancestry groups can serve as a global reference of worldwide populations, with a handful of applications. As an example application here, we use allele frequencies derived from these ancestry groups to effectively measure diversity from summary statistics of any genetic dataset. Measuring genetic diversity is an important problem because increasing genetic diversity is key to making new genetic discoveries, while also being a major source of confounding to be aware of in genetics studies.
\end{abstract}


Several projects have focused on providing genetic data from diverse populations, such as the HapMap project, the 1000 genomes project $(1 \mathrm{KG})$, the Simons genome diversity project, and the human genome diversity project (International HapMap 3 Consortium et al. 2010; 1000 Genomes Project Consortium et al. 2015; Mallick et al. 2016; Bergström et al. 2020). However, these datasets do not contain many individuals per population and therefore are not large enough for some purposes, such as accurately estimating allele frequencies for diverse worldwide populations. The UK Biobank (UKBB) project is a prospective cohort study with deep genetic and phenotypic data collected on almost 500,000 individuals from across the United Kingdom. Despite being a cohort from the UK, this dataset is so large that it includes individuals that were born in all four corners of the world. Here we carefully use information on self-reported ancestry, country of birth, and genetic similarity to define 21 distinct ancestry groups from the UK Biobank to be used as global reference populations. This includes nine groups with genetic ancestries from Europe, four from Africa, three from South Asia, three from East Asia, one from the Middle East, and one from South America (Table 11. The detailed procedure used to define these groups is presented in the Supplementary Materials.

As an example application of these groups, we propose to estimate global ancestry proportions from a cohort based on its allele frequencies only (i.e. summary statistics). Arriaga-MacKenzie et al. (2021) previously proposed method Summix, which finds the convex combination of ancestry proportions $\alpha_{k}$ (positive and sum to 1) which minimizes the following problem: $\sum_{j=1}^{M}\left(f_{j}^{(0)}-\sum_{k=1}^{K} \alpha_{k} f_{j}^{(k)}\right)^{2}$, where $M$ is the number of variants, $K$ the number of reference populations, $f_{j}^{(k)}$ is the frequency of variant $j$ in population $k$, and $f_{j}^{(0)}$ is the frequency of variant $j$ in the cohort of interest. In Arriaga-MacKenzie et al. (2021), they used the five continental $1 \mathrm{KG}$ populations as reference. Here we provide reference allele frequencies for 5,816,590 genetic variants across 21 diverse ancestry groups (which are later merged in 18 groups in table 11. Moreover, we rely on the projection of our reference allele frequencies onto the PCA (principal component analysis) space computed from the corresponding UKBB (and $1 \mathrm{KG}$ ) individuals. This is described in more details in the Supplementary Materials. Finding the ancestry proportions in the PCA space (rather than using the allele frequencies directly) provides more power to distinguish between close populations. This enables us to use more reference populations (18) in order to get a more fine-grained measure of genetic diversity. We implement this method in function snp_ancestry_summary in our R package bigsnpr (Privé et al. 2018), relying on R package quadprog (Turlach et al.2019) for solving this quadratic programming optimization problem.

We download several GWAS summary statistics for which allele frequencies are reported, and apply this method to them. We first apply function snp_ancestry_summary to more homogeneous samples as an empirical validation; when applying it to the Biobank Japan (Japanese cohort), FinnGen (Finnish), a Peruvian cohort, a Qatari cohort and Sub-Saharan African cohort, the ancestry proportions obtained match expectations (Table 1). When comparing our estimates with reported ancestries for more diverse cohorts, for example PAGE is composed of 44.6\% Hispanic-Latinos, 34.7\% African-Americans, 9.4\% Asians, 7.9\% Native Hawaiians and 3.4\% of some other ancestries (self-reported), whereas our estimates are of $25.4 \%$ South American, 22.6\% European (including 15.8\% from South-West Europe), 34.1\% African, 2.7\% South Asian, 10.6\% East Asian, and 4.6\% Filipino. GWAS summary statistics from either European ancestries or more diverse ancestries all have a substantial proportion estimated from European ancestry groups, while ancestries from other continents are still largely underrepresented (Table 1 ). 


\begin{tabular}{|c|c|c|c|c|c|c|c|c|c|c|c|c|c|c|c|c|}
\hline Ancestry group & $\mathrm{N}$ & BBJ & FinnGen & Perú & Qatar & Africa & GERA & PAGE & $\mathrm{BrCa}$ & PrCa & CAD & body fat & covid & eczema & epilepsy & urate \\
\hline Africa (West) & 735 & & & & & 30 & 1.9 & 27.7 & 0.3 & 0.3 & 2.2 & 0.7 & 4 & 0.2 & 0.7 & 2.2 \\
\hline Africa (South) & 449 & & & & & 70 & 0.9 & 5.9 & 0.2 & & 1.2 & 0.3 & 1.2 & 0.5 & 0.3 & 3.5 \\
\hline Africa (East) & 276 & & & & 13 & & & & & & 0.1 & & 0.3 & & & 1.9 \\
\hline Africa (North) & 268 & & & & 22 & & & 0.5 & & & & & 0.1 & & & \\
\hline Middle East & 523 & & & & 64.6 & & & & & & & 0.2 & & & & 1.3 \\
\hline Ashkenazi & 1975 & & & & & & 4.4 & 0.5 & 0.2 & 1.8 & 0.4 & 0.8 & 0.4 & 0.6 & 1.8 & \\
\hline Italy & 345 & & & & & & 4.6 & & 3.1 & 1.2 & 9.7 & 5.5 & & & 3.4 & 0.8 \\
\hline Europe (East) & 667 & & & & & & 10.5 & & 6.9 & 11.3 & 10.5 & 11.4 & 13.2 & 11.7 & 13.9 & 10.8 \\
\hline Finland & $143(+99)$ & & 100 & & & & 2.4 & 0.7 & 9.7 & 13 & 5.9 & 8.8 & 14.8 & 12.8 & 6.5 & 2 \\
\hline Europe (North West) & 4416 & & & & & & 59.9 & 5.6 & 68.5 & 64.5 & 51.8 & 59.8 & 61.4 & 70.9 & 68 & 46 \\
\hline Europe (South West) & 603 & & & & & & 3.5 & 15.8 & 4.7 & 4.5 & & & & 2.1 & 2.1 & \\
\hline South America & $473(+84)$ & & & 100 & & & 4.6 & 25.4 & 1.5 & 0.8 & 1.6 & 0.4 & 1.8 & 0.5 & & \\
\hline Sri Lanka & 372 & & & & 0.4 & & 0.4 & & 3.4 & 1.7 & 4.7 & 4.2 & 1.8 & & & 1.9 \\
\hline Pakistan & 400 & & & & & & & 1.1 & & & 7 & 4 & & & & \\
\hline Bangladesh & $223(+86)$ & & & & & & & 1.6 & & & & & & & & \\
\hline Asia (East) & 961 & & & & & & 3.5 & 1.2 & 1.2 & 0.7 & 2.5 & 1.2 & 0.1 & 0.1 & 3.1 & \\
\hline Japan & $240(+104)$ & 100 & & & & & 2.2 & 9.4 & 0.4 & & 2.4 & 2.8 & 0.7 & 0.7 & 0.3 & 29.8 \\
\hline Philippines & 295 & & & & & & 1.5 & 4.6 & & & & & 0.2 & & & \\
\hline
\end{tabular}

Table 1: Reference populations with their size (N), and corresponding ancestry proportions (in \%) from several GWAS datasets. Note that "Ireland", "United Kingdom" and "Scandinavia" are merged as a single "Europe (North West)" group. Similarly, "Europe (North East)" and "Europe (South East)" are merged as a single "Europe (East)" group. Citations for the allele frequencies used: the Biobank Japan (BBJ, Sakaue et al. (2021)), FinnGen (https://r5.finngen.fi/), GWAS in Peruvians (Asgari et al. 2020), GWAS in Qataris (Thareja et al. 2021), GWAS in Sub-Saharan Africans (Africa, Chen et al. (2019)), GERA (Hoffmann et al. 2018), PAGE (Wojcik et al. 2019), breast cancer (BrCa, Michailidou et al. (2017)), prostate cancer (PrCa, Schumacher et al. (2018)), coronary artery disease (CAD, Nikpay et al. (2015)), body fat percentage (Lu et al. 2016), COVID-19 (The COVID-19 Host Genetics Initiative and Ganna 2021), eczema (Paternoster et al. 2015), epilepsy (The International League Against Epilepsy Consortium on Complex Epilepsies 2018), and serum urate (Tin et al. 2019). Several of these GWAS summary statistics have been downloaded through the NHGRI-EBI GWAS Catalog (MacArthur et al. 2017).

Here we identify an unprecedentedly large and diverse set of ancestry groups within a single cohort, the UK Biobank. Using allele frequencies derived from these ancestry groups (and the individual-level data for computing the PCs), we show how to effectively measure diversity from GWAS summary statistics reporting allele frequencies. Measuring genetic diversity is an important problem because increasing genetic diversity is key to making new genetic discoveries, while also being a major source of confounding to be aware of in genetics studies. Our work has limitations though. First, it is not fully clear whether we can capture any ancestry, e.g. Native Hawaiians in the PAGE study; it seems that they are partly captured by the "Philippines" ancestry group we define. Second, with the 21 ancestry groups we define, we think we are capturing a large proportion of the genetic diversity in Europe, but we may still be missing more fine-grained diversity in other continents. Third, when we use the allele frequencies reported in the GWAS summary statistics, it is not clear whether they were computed from all individuals (i.e. before performing any quality control and filtering), and, for meta-analyses of binary traits, whether they were computed as a weighted average of total or effective sample sizes. Despite these limitations, we envision that the ancestry groups we define here will have many useful applications. The presented method that uses these groups could e.g. be used to automatically report ancestry proportions in the GWAS Catalog (MacArthur et al. 2017). These ancestry groups could also be used for assigning ancestry in other cohorts using the PC projection from this study (Privé et al. 2021), assessing portability of polygenic scores (Privé et al. 2021), or deriving linkage disequilibrium (LD) references matching GWAS summary statistics from diverse ancestries. 


\section{Software and code availability}

The newest version of R package bigsnpr can be installed from GitHub (see https://github.com/ privefl/bigsnpr). Refer to the help page of function snp_ancestry_summary for how to use it. The set of reference allele frequencies for 5,816,590 genetic variants across 21 diverse ancestry groups defined here can be downloaded at https://figshare.com/ndownloader/files/31620968 and PC loadings for all variants across $16 \mathrm{PCs}$ at https://figshare.com/ndownloader/files/ 31620953. All code used for this paper is available at https://github.com/privefl/freq-ancestry/ tree/main/code. We have extensively used R packages bigstatsr and bigsnpr (Privé et al. 2018) for analyzing large genetic data, packages from the future framework (Bengtsson 2021) for easy scheduling and parallelization of analyses on the HPC cluster, and packages from the tidyverse suite (Wickham et al.|2019) for shaping and visualizing results.

\section{Acknowledgements}

F.P. is supported by the Danish National Research Foundation (Niels Bohr Professorship to John McGrath) and by a Lundbeck Foundation Fellowship (R335-2019-2339 to Bjarni J. Vilhjálmsson). The author thanks GenomeDK and Aarhus University for providing computational resources and support that contributed to these research results. This research has been conducted using the UK Biobank Resource under Application Number 58024.

\section{Declaration of Interests}

The author declares no competing interests. 


\section{References}

1000 Genomes Project Consortium et al. (2015). A global reference for human genetic variation. Nature, 526(7571), 68.

Arriaga-MacKenzie, I. S., Matesi, G., Chen, S., Ronco, A., Marker, K. M., Hall, J. R., Scherenberg, R., KhajehSharafabadi, M., Wu, Y., Gignoux, C. R., et al. (2021). Summix: A method for detecting and adjusting for population structure in genetic summary data. The American Journal of Human Genetics.

Asgari, S., Luo, Y., Akbari, A., Belbin, G. M., Li, X., Harris, D. N., Selig, M., Bartell, E., Calderon, R., Slowikowski, K., et al. (2020). A positively selected FBN1 missense variant reduces height in peruvian individuals. Nature, 582(7811), 234-239.

Bengtsson, H. (2021). A Unifying Framework for Parallel and Distributed Processing in R using Futures. The $R$ Journal.

Bergström, A., McCarthy, S. A., Hui, R., Almarri, M. A., Ayub, Q., Danecek, P., Chen, Y., Felkel, S., Hallast, P., Kamm, J., et al. (2020). Insights into human genetic variation and population history from 929 diverse genomes. Science, 367(6484).

Chen, J., Sun, M., Adeyemo, A., Pirie, F., Carstensen, T., Pomilla, C., Doumatey, A. P., Chen, G., Young, E. H., Sandhu, M., et al. (2019). Genome-wide association study of type 2 diabetes in Africa. Diabetologia, 62(7), 1204-1211.

Hoffmann, T. J., Theusch, E., Haldar, T., Ranatunga, D. K., Jorgenson, E., Medina, M. W., Kvale, M. N., Kwok, P.-Y., Schaefer, C., Krauss, R. M., et al. (2018). A large electronic-health-record-based genome-wide study of serum lipids. Nature Genetics, 50(3), 401-413.

International HapMap 3 Consortium et al. (2010). Integrating common and rare genetic variation in diverse human populations. Nature, $\mathbf{4 6 7 ( 7 3 1 1 ) , 5 2 .}$

Lu, Y., Day, F. R., Gustafsson, S., Buchkovich, M. L., Na, J., Bataille, V., Cousminer, D. L., Dastani, Z., Drong, A. W., Esko, T., et al. (2016). New loci for body fat percentage reveal link between adiposity and cardiometabolic disease risk. Nature Communications, 7(1), 1-15.

MacArthur, J., Bowler, E., Cerezo, M., Gil, L., Hall, P., Hastings, E., Junkins, H., McMahon, A., Milano, A., Morales, J., et al. (2017). The new NHGRI-EBI Catalog of published genome-wide association studies (GWAS Catalog). Nucleic Acids Research, 45(D1), D896-D901.

Mallick, S., Li, H., Lipson, M., Mathieson, I., Gymrek, M., Racimo, F., Zhao, M., Chennagiri, N., Nordenfelt, S., Tandon, A., et al. (2016). The simons genome diversity project: 300 genomes from 142 diverse populations. Nature, 538(7624), 201-206.

Michailidou, K., Lindström, S., Dennis, J., Beesley, J., Hui, S., Kar, S., Lemaçon, A., Soucy, P., Glubb, D., Rostamianfar, A., et al. (2017). Association analysis identifies 65 new breast cancer risk loci. Nature, 551(7678), 92.

Nikpay, M., Goel, A., Won, H.-H., Hall, L. M., Willenborg, C., Kanoni, S., Saleheen, D., Kyriakou, T., Nelson, C. P., Hopewell, J. C., et al. (2015). A comprehensive 1000 genomes-based genome-wide association meta-analysis of coronary artery disease. Nature Genetics, 47(10), 1121. 
Paternoster, L., Standl, M., Waage, J., Baurecht, H., Hotze, M., Strachan, D. P., Curtin, J. A., Bønnelykke, K., Tian, C., Takahashi, A., et al. (2015). Multi-ethnic genome-wide association study of 21,000 cases and 95,000 controls identifies new risk loci for atopic dermatitis. Nature Genetics, 47(12), 1449.

Privé, F., Aschard, H., Ziyatdinov, A., and Blum, M. G. B. (2018). Efficient analysis of large-scale genome-wide data with two R packages: bigstatsr and bigsnpr. Bioinformatics, 34(16), 2781-2787.

Privé, F., Aschard, H., Carmi, S., Folkersen, L., Hoggart, C., O’Reilly, P. F., and Vilhjálmsson, B. J. (2021). Highresolution portability of 245 polygenic scores when derived and applied in the same cohort. medRxiv.

Sakaue, S., Kanai, M., Tanigawa, Y., Karjalainen, J., Kurki, M., Koshiba, S., Narita, A., Konuma, T., Yamamoto, K., Akiyama, M., et al. (2021). A cross-population atlas of genetic associations for 220 human phenotypes. Nature Genetics, pages 1-10.

Schumacher, F. R., Al Olama, A. A., Berndt, S. I., Benlloch, S., Ahmed, M., Saunders, E. J., Dadaev, T., Leongamornlert, D., Anokian, E., Cieza-Borrella, C., et al. (2018). Association analyses of more than 140,000 men identify 63 new prostate cancer susceptibility loci. Nature Genetics, 50(7), 928.

Thareja, G., Al-Sarraj, Y., Belkadi, A., Almotawa, M., Suhre, K., and Albagha, O. M. (2021). Whole genome sequencing in the middle eastern Qatari population identifies genetic associations with 45 clinically relevant traits. Nature Communications, 12(1), 1-10.

The COVID-19 Host Genetics Initiative and Ganna, A. (2021). Mapping the human genetic architecture of covid-19 by worldwide meta-analysis. MedRxiv.

The International League Against Epilepsy Consortium on Complex Epilepsies (2018). Genome-wide mega-analysis identifies 16 loci and highlights diverse biological mechanisms in the common epilepsies. Nature Communications, 9.

Tin, A., Marten, J., Kuhns, V. L. H., Li, Y., Wuttke, M., Kirsten, H., Sieber, K. B., Qiu, C., Gorski, M., Yu, Z., et al. (2019). Target genes, variants, tissues and transcriptional pathways influencing human serum urate levels. Nature Genetics, 51(10), 1459-1474.

Turlach, B. A., Weingessel, A., and Moler, C. (2019). quadprog: Functions to Solve Quadratic Programming Problems. R package version 1.5-8.

Wickham, H., Averick, M., Bryan, J., Chang, W., McGowan, L. D., François, R., Grolemund, G., Hayes, A., Henry, L., Hester, J., et al. (2019). Welcome to the tidyverse. Journal of Open Source Software, 4(43), 1686.

Wojcik, G. L., Graff, M., Nishimura, K. K., Tao, R., Haessler, J., Gignoux, C. R., Highland, H. M., Patel, Y. M., Sorokin, E. P., Avery, C. L., et al. (2019). Genetic analyses of diverse populations improves discovery for complex traits. Nature, 570(7762), 514-518. 